\title{
Feasibility study of an electro-optical absorption based method for the measurement of endogenous chemicals in living and ex-vivo tissues
}

\author{
Francesco Crespi* \\ Department of Biology, GSK, Verona, Italy
}

\section{Background}

In general, in vivo analysis of neurotransmitter activities are based upon electro-chemical methods that require Invasive implantation of electrodes or probes within the animal's brain. In 1990, a completely new approach to selectively monitor neurotransmitters based upon analysis of their own fluorescence using LASER induced chemical excitation of their molecules has been introduced [1]. In particular, this approach has demonstrated that the spectral intensity distribution, the decay time and the relative quantum yield of a neurotransmitter such as serotonin can be selectively evaluated by means of a fiber optic and LASER spectroscopy in vitro $[2,3]$.

The demand that aroused since this preliminary work was about the possibility of the application of such a LASER based optical technique for in vivo analysis by means of fiber optics. In particular, the main question was about the feasibility of applying such a technique without entering the brain (or other) tissue: i.e. via the application of the fiber optic just in contact with the surface of the head or the skin and/or other parts of the body. Consequently, non-invasive in vivo infrared laser spectroscopy (NIRS) to analyses endogenous oxy-haemoglobin, deoxy-haemoglobin, and blood volume in the rat central nervous system (CNS) has been achieved [4]. This methodology allows real time non-invasive monitoring of brain penetration of chemicals [5] as well as in vivo analysis of the effects of drugs of abuse upon rat brain oxygenation [6]. It is also able to monitor parallel alteration of brain metabolism following alcohol intake in rodents and man [7] thus permitting to propose the NIRS methodology as translational strategy from preclinical to clinical investigations in particular in the CNS.

Actually, the feasibility of analyzing other parts of the body is under evaluation. In particular, the project is to evaluate the feasibility of an in vivo optical method for the concentration measurement of endogenous chemicals in the tissues of laboratory rats. The possible applications are not only the measurement of tissue oxygenation, but also and specifically the analysis of chemicals (i.e. serotonin, dopamine, GABA, etc.) in brain tissues or in blood, i.e. compounds that are involved in drug-dependency, anxiety-depression, brain diseases studies.

\section{Principle}

The principle of this technique is the detection of the optical absorption, by the tissue under test, of radiation at given wavelengths. Illumination of the tissue and detection of diffused radiation can occur by means of an optical fiber or, to reduce invasiveness, across the skin or possibly a thin bone layer.
In Figure $1, \lambda 1$ is the peak absorption wavelength of the target substance, whereas $\lambda 2$ is a low absorption wavelength. Then, one can assume that the tissue background shows a negligible difference in the absorption between $\lambda 1$ and $\lambda 2$. In that case the concentration of the target substance will be approximately,

$$
c \cong K \frac{A\left(\lambda_{1}\right)-A\left(\lambda_{2}\right)}{A\left(\lambda_{2}\right)}
$$

where $A \lambda 1$ and $A \lambda 2$ are the absorption values of the tissue under measure respectively at $\lambda 1$ and $\lambda 2$, and $\mathrm{K}$ is a constant of proportionality.

The technique proposed works with wavelengths $(400-1300 \mathrm{~nm})$ that show a longer absorption depth, allowing for deeper non-invasive inspection in living tissues. The main feature is that the measurement setup is based on two monochromatic optical sources at $\lambda 1$ and $\lambda 2$, a fiber optic system and a photo-detection network (Figure 2). The same optical fiber carries radiation of both wavelengths and collects scattered power, redirecting it to two separate photo-detectors, each one sensitive to one of the wavelengths. A single photo-detector can be used, providing that the two sources are pulsed in turn. The tip of

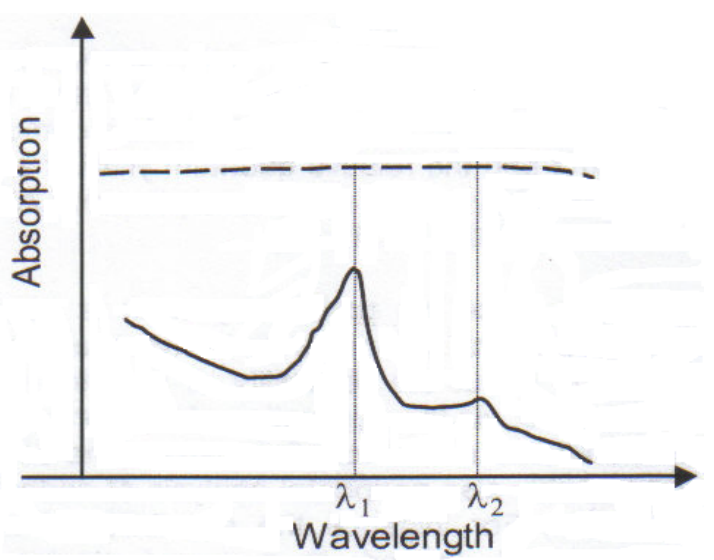

Figure 1. Optical absorption spectrum of the target substance (continuous line) and of the background (dashed line)

${ }^{\star}$ Correspondence to: Francesco Crespi, Department of Biology, GSK, Verona, Italy, E-mail: fm.crespi@libero.it

Received: February 04, 2019; Accepted: February 22, 2019; Published: February 25,2019 


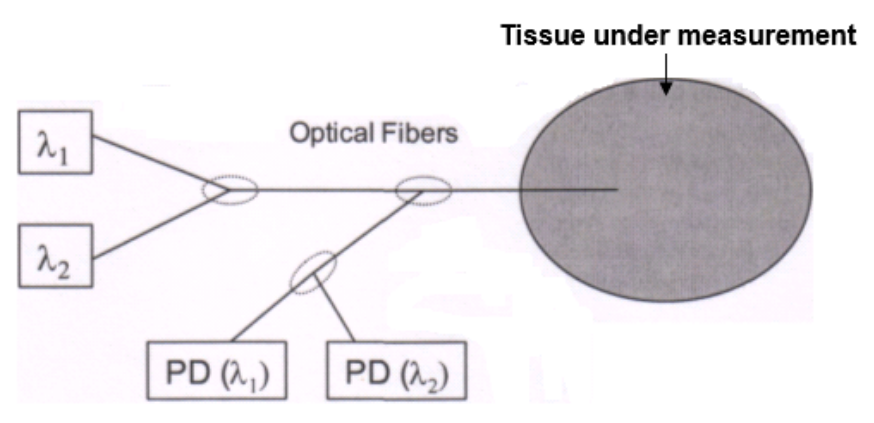

Figure 2. Block scheme of the measurement setup. $\lambda 1$ and $\lambda 2$ : monochromatic sources. PD $(\lambda 1)$ and PD $(\lambda 2)$ : photodetectors

the optical fiber, working as a probe, has to be set in contact with the sample tissue.

The novelty of the method proposed is therefore based on double monochromatic illumination and detection, characteristic that offers higher resolution than a conventional fluorimetric technique, in which energy gets spread on a broad spectrum. In addition, fluorescence methods rely upon near-UV illumination (close to $300 \mathrm{~nm}$ ), which has a very short absorption depth in living tissues.

\section{Project tasks}

To evaluate the feasibility of the method, several tasks have to be undergone.

- The first issue to be addressed is the reconstruction of the absorption spectrum of the target substances (serotonin, dopamine, GABA or other endogenous chemicals of interest) in a wavelength range from visible to near infrared $(400-1300 \mathrm{~nm})$. This is the range in which most sources and optics come at hand. For each substance, we have to identify the two wavelengths $\lambda 1$ and $\lambda 2$ where the optical absorption is respectively strong and weak.

- Then, the absorption spectra of most common background tissues, like the brain, plasma or skin of laboratory rats, are to be retrieved. At this point, one has to check that the background absorption is almost uniform between $\lambda 1$ and $\lambda 2$. It is also preferred that the background absorption is low at the wavelength of interest so that the concentration of the target substance should not to be masked by the background.

- Given the spectra of the target substance and the optical absorption of the background, one can estimate the amount of optical power needed. The lower the absorption of the tissue, the deeper the measurement can go into the sample. Two cases are to be evaluated in detail: 1) analysis of neurotransmitters in the brain and 2) measurement on peripheral tissues and fluids such as plasma. Measurement depth depends upon the absorption length of the radiation used. In addition, this technique has to be tested from across a barrier layer, being it the skull bone for brain analysis or the skin for blood analysis.

- A full design of the measurement set-up has to be devised. The optical sources can be chosen between lasers, leds or filtered lamps depending upon the power and wavelength needed. A detailed configuration of the coupling optics, the fiber system and of the photo-detection network has to be designed.

- Finally, this method has to be compared to the existing techniques like multi-photon excitation or fluorescence detection, whose typical results are found in literature. The comparison has to be carried in terms of optical power required, measurement resolution, complexity and cost of the set-up.

\section{Outcome}

The results of absorption spectra measurements for different target substances and backgrounds will be analyzed. Then, the best-choice measurement scenario, in terms of target chemical and background tissue, will be identified. This will possibly permit central (CNS) and eventually also peripheral Non-Invasive analysis of endogenous chemicals without need for any surgical preparation, therefore allowing measurements in real time and in physiological conditions. For instance, the possibility of monitoring specific blood components could also be analyzed. Indeed, in a previous electrochemical work it has been demonstrated that peripheral serotonin monitored in platelet rich plasma mimic cerebral extracellular serotonin levels and that these peripheral levels are reduced as well as the CNS levels in animal models of depression [8]. Low levels of central serotonin have been related to the state of depression. Thus, the analysis of peripheral serotonin with the "LASER" methodology would allow the study of the, "mood" in real time and in absolutely normal physiological state. Finally, the success of such an approach will be of value for in vivo laboratory testing and also will lead the way of future applications in diagnostics and therapeutics.

\section{References}

1. Crespi F (1990) In vivo voltammetry with micro-biosensors for analysis of neurotransmitter release and metabolism. J Neurosci Methods 34: 53-65. [Crossref]

2. Crespi F, Dalessandro D, Annovazzi-Lodi V, Norgia M (2004) In vivo voltammetry from wire to wireless measurements. J Neurosci Methods 140: 153-161. [Crossref]

3. Crespi F, Croce AC, Fiorani S, Masala B, Bottiroli G, et al. (2004) Autofluorescence Spectrofluorometry of central nervous system (CNS) neuromediators. Lasers Surg Med 34: 39-47. [Crossref]

4. Crespi F (2007) Near-infrared spectroscopy (NIRS): a non-invasive in vivo methodology for analysis of brain vascular and metabolic activities in real time in rodents. Curren Vas Pharmacol 5: 305-321. [Crossref]

5. Crespi F, Cottini S, Bandera A, Donini M, Rovati L, et al. (2016) In vivo real time noninvasive monitoring of brain penetration of chemicals with near-infrared spectroscopy: concomitant PK/PD analysis. J Neurosci Methods 258: 79-86. [Crossref]

6. Crespi F, Donini M, Bandera A, Congestri, F, Formenti, F, et al. (2006) Near infrared oxymeter biosensor-prototype for non-invasive in vivo analysis of rat brain oxygenation: effects of drugs of abuse. J Optics: Pure Appl Optics 8: 528-534.

7. Crespi F, Congestri F, Donini M (2018) Translational NIRS: Parallel Alteration of Brain Metabolism Following Alcohol Intake in Rodents and Man. J Neurodegener Disord 2: $22-31$.

8. Bianchi M, Moser C, Lazzarini C, Vecchiato E, Crespi F, et al. (2002) Forced swimming test and fluoxetine treatment: in vivo evidence that Periphal 5-HT in rat platelet-rich plasma mirrors cerebral extracellular 5-HT levels, whilst 5-HT in isolated platelets mirrors neuronal 5-HT changes. Exp Brain Res 143: 191-197. [Crossref]

Copyright: (C2019 Crespi F. This is an open-access article distributed under the terms of the Creative Commons Attribution License, which permits unrestricted use, distribution, and reproduction in any medium, provided the original author and source are credited. 